\title{
Purification d'air par photocatalyse hétérogène
}

\author{
P. Pichat
}

\author{
Photocatalyse, Catalyse et Environnement, CNRS UMR « IFoS », Ecole Centrale de Lyon, \\ BP. 163, 69131 Ecully cedex, France
}

\begin{abstract}
Résumé : La photocatalyse hétérogène repose sur l'activation d'un semiconducteur, le plus souvent $\mathrm{TiO}_{2}$, par des radiations d'énergie au moins égale à celle de la largeur de la bande interdite. Les changements électroniques provoqués par cette activation conduisent, en présence d'air, à la création de radicaux oxygénés. Ces radicaux attaquent les composés organiques adsorbés et les dégradent. A partir d'évaluations effectuées avec un photoréacteur de laboratoire, validées par des mesures réalisées avec un purificateur-prototype dans une pièce ordinaire, nous montrons que la photocatalyse est une méthode appropriée pour purifier l'air dans l'habitat (ou d'autres lieux confinés). Par ailleurs, de bons résultats ont été obtenus pour le traitement photocatalytique d'air ayant servi à extraire des hydrocarbures aliphatiques chlorés légers de sols ou d'eaux.
\end{abstract}

\section{INTRODUCTION}

La photocatalyse hétérogène $[1,2]$ est fondée sur l'absorption de rayonnement excitateurs, le plus souvent ultraviolets, par un semiconducteur. $\mathrm{TiO}_{2}$ fournit les photocatalyseurs les plus actifs. L'excitation photonique de $\mathrm{TiO}_{2}$ (largeur de la bande interdite proche de $3 \mathrm{eV}$ ou $400 \mathrm{~nm}$ ) revient à créer des paires électrons-trous positifs, c'est-à-dire un système oxydo-réducteur. Les électrons (e) ou les trous $\left(\mathrm{h}^{+}\right)$ peuvent être captés, respectivement, par $\mathrm{O}_{2}$ ou la vapeur d'eau, les groupes $\mathrm{OH}$ superficiels et certains polluants pour former des radicaux ou des ions-radicaux (Fig.1). Ces radicaux se transforment, réagissent entre eux ou attaquent les composés organiques adsorbés sur $\mathrm{TiO}_{2}$ qui ainsi se dégradent.

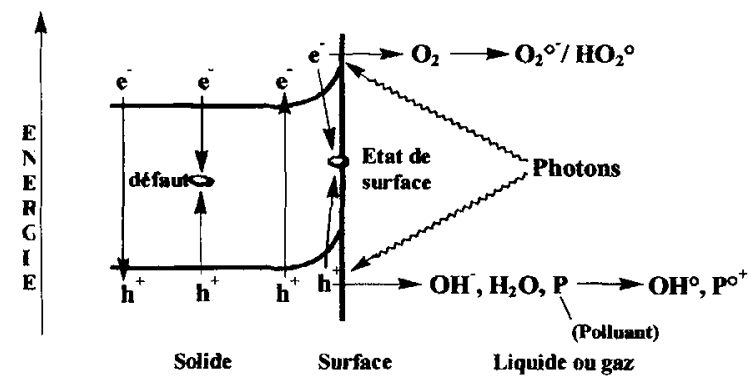

Figure 1: Schéma montrant l'excitation d'un semiconducteur de type $\mathrm{n}$, tel que $\mathrm{TiO}_{2}$, les recombinaisons des porteurs de charge et les transferts électroniques interfaciaux aux groupes superficiels et aux espèces adsorbées.

Plusieurs applications $[1,2]$ découlent de ces possibilités de dégradation : parmi elles, les dépollutions de milieux gazeux ou liquides, notamment de l'air — aussi bien l'air ambiant intérieur que les effluents et de l'eau. La matière organique, y compris celle des micro-organismes [2-4], est oxydée et finalement complètement minéralisée si les temps de contact entre les produits formés de façon intermédiaire et $\mathrm{TiO}_{2}$ sont suffisamment longs. Certains composés minéraux oxydables ou réductibles sont également transformés chimiquement. Si l'on recouvre d'une mince couche de $\mathrm{TiO}_{2}$ la surface de matériaux non dégradables par photocatalyse (matériaux inorganiques ou polymères ne comportant que des liaisons $\mathrm{C}-\mathrm{F}$ ) les composés organiques qui se déposent sur ces matériaux sont progressivement dégradés sous l'effet des radiations UV solaires: ces matériaux s'auto-nettoient [5]. Le problème principal est d'obtenir une adhérence de la couche de $\mathrm{TiO}_{2}$ suffisante compte tenu du type d'emploi du matériau. 
L'émergence de ces applications - ajoutées à celles concernant la production d'énergie électrique [6] et les synthèses organiques $[7,8]$ - a entraîné une croissance très forte des travaux dans le domaine de la photocatalyse (environ 300 publications et 400 brevets pour la seule année 1999) [2].

Le traitement d'air par photocatalyse au contact de $\mathrm{TiO}_{2}$ offre divers avantages.

- Il n'est pas nécessaire d'ajouter de composé chimique, contrairement au cas du traitement des odeurs par des agents masquants ou neutralisants ou encore à la purification par ozonation.

- $\mathrm{TiO}_{2}$ est un composé non toxique, peu coûteux ; de plus, il est stable et, en principe, il s'autorégénère en cours d'usage, c'est-à-dire que les produits des polluants organiques initiaux qui s'accumuleraient transitoirement (pollution intermittente) à sa surface sont à leur tour minéralisés.

- Grâce au phénomène d'adsorption qui concentre les polluants à la surface de $\mathrm{TiO}_{2}$ où sont produits les espèces actives, la photocatalyse est bien adaptée à l'épuration/déodorisation d'air ambiant ou d'effluents contenant seulement des traces de polluants toxiques et/ou malodorants.

- Contrairement aux technologies fondées exclusivement sur les phénomènes d'adsorption ou d'absorption qui transfèrent les polluants, ce qui entraîne des traitements supplémentaires, la photocatalyse peut minéraliser totalement les polluants organiques ou du moins permet d'atteindre des concentrations de tous les composés - initiaux ou produits - suffisamment basses.

- C'est une méthode susceptible de dégrader tous les polluants organiques, mis à part les chlorofluorocarbones et le cycle $s$-triazine (1,3,5-triazabenzène).

En revanche, les vitesses des transformations chimiques sont limitées par la vitesse des recombinaisons entre les électrons et les trous, soit au sein de $\mathrm{TiO}_{2}$, soit à sa surface (Fig. 1), notamment en raison de l'existence de défauts structuraux et de la présence de traces de cations étrangers en position de substitution ou interstitielle. Cette vitesse de recombinaison, difficilement maitrisable, limite les domaines d'application de la photocatalyse.

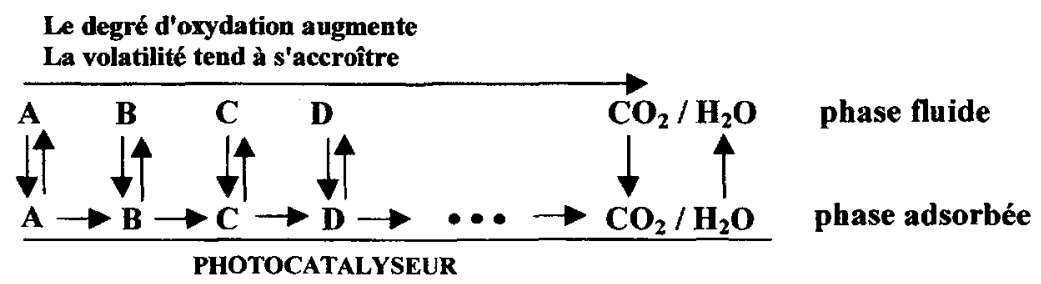

Figure 2. Schéma simplifié (absence de réactions parallèles) montrant la transformation photocatalytique progressive d'un polluant organique $\mathrm{A}$ en produits $\mathrm{B}, \mathrm{C}, \mathrm{D}$, etc. jusqu'à la minéralisation complète.

\section{EFFICACITE DU TRAITEMENT PHOTOCATALYTIQUE (réacteur de laboratoire)}

De nombreux facteurs déterminent la vitesse d'élimination photocatalytique de polluants, notamment la nature du matériau "photocatalytique" (type de $\mathrm{TiO}_{2}$, caractéristiques du support, méthode de dépôt) et, bien entendu, les dimensions et la conception du réacteur photocatalytique : qualité de l'irradiation et du contact air à traiter-photocatalyseur. La Fig. 2 illustre l'influence des dimensions du réacteur, en montrant que pour un réacteur cylindrique où l'air (débit et concentration en polluant $A$ fixés) passe tangentiellement au matériau photocatalytique et/ou traverse successivement des couches de ce matériau, une faible longueur du réacteur permettra la transformation de A principalement en ses premiers produits, tandis qu'une grande longueur permettra d'atteindre la minéralisation complète. Par ailleurs, le nombre de paires électron-trou créées par les photons atteignant $\mathrm{TiO}_{2}$ dépend, pour un flux irradiant donné, de l'énergie hc/ $\lambda$ d'un photon, ainsi que de l'absorption $(\lambda<400 \mathrm{~nm})$, de la réflexion et de la diffusion des photons par les grains de $\mathrm{TiO}_{2}$ - et, éventuellement, par le liant employé pour les fixer sur un support dans le domaine spectral choisi. Des essais comparatifs de lampes UV sont nécessaires pour chaque type de dispositif et de matériau, les calculs s'avérant trop complexes.

Pour des réacteurs "bien conçus" et pour un "bon" matériau photocatalytique, des expériences de laboratoire sont utiles pour déterminer un ordre de grandeur de cette vitesse d'élimination et en tirer des conclusions pratiques quant aux possibilités et limites de ce type de traitement. Nous avons employé un réacteur photocatalytique cylindrique [9] contenant une lampe UV, émettant à $365 \mathrm{~nm}$, entourée d'environ 
$1300 \mathrm{~cm}^{2}$ d'une toile en fibres de verre recouverte de $\mathrm{TiO}_{2}$ (Matrix Photocatalytic, Inc.), avec une puissance électrique correspondant environ au $1 / 5$ de la puissance considérée comme raisonnable pour améliorer la qualité de l'air dans une pièce. L'inconvénient de ce réacteur est que les débits d'air maximaux sont seulement de $50 \mathrm{~L} \mathrm{~h}^{41}$ alors que pour un réacteur destiné à purifier l'air ambiant ou un effluent gazeux, le débit total sera au moins 1000 fois plus grand, donc le temps de passage 1000 fois plus court, d'où la nécessité de valider les résultats avec un prototype fonctionnant avec ces débits plus élevés.

Afin de conférer un caractère assez général à cette évaluation de l'ordre de grandeur de l'élimination photocatalytique par unités de temps et de puissance électrique, nous avons choisi des polluants de structure chimique très différente [9]. Pour des débits d'air ou de dioxygène sec compris entre 25 et $50 \mathrm{~L} \mathrm{~h}^{-1}$ et des concentrations de 50 à $2000 \mathrm{ppmv}$, nous avons trouvé des vitesses d'élimination allant de 5 à $15 \mu \mathrm{mol}$ par Wh consommé par la lampe UV. Nos propres résultats et ceux de la bibliographie [2] montrent que l'humidité relative et la température au voisinage des conditions ambiantes ne changent pas l'ordre de grandeur de la vitesse d'élimination photocatalytique. Cet ordre de grandeur devrait aussi rester valable pour un grand nombre de polluants (exceptés, notamment, le méthanal, très instable, et certains alcènes et alcanes chlorés pour lesquels des résultats contradictoires ont été observés).

\section{PURIFICATION D'AIR DANS UNE PIECE}

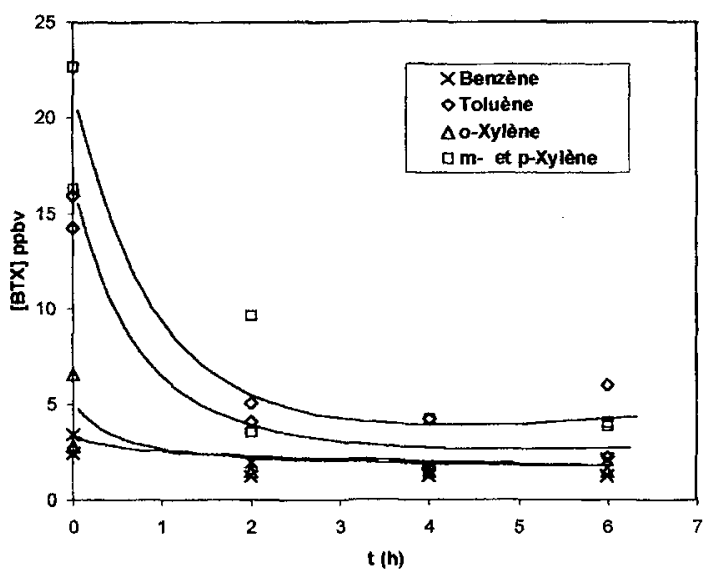

Figure 3: Variations des concentrations des hydrocarbures indiqués dans l'air d'une pièce : avant mise en fonctionnement des lampes UV du purificateur $(\mathrm{t}=\mathrm{o}$; les mesures montrant les variations de la composition de l'atmosphère de la pièce pendant quelques heures) et lors du traitement par le purificateur $(t=2 ; 4 ; 6 \mathrm{~h})$.

L'ordre de grandeur de $10 \mu \mathrm{mol} \mathrm{Wh}^{-1}$ peut être utilisé pour déterminer a priori si un purificateur d'air individuel employant le même type de lampe UV et un matériau recouvert de $\mathrm{TiO}_{2}$ similaire peut être efficace. En effet, $10 \mu \mathrm{mol}$ correspondent à une concentration de $10 \mathrm{ppbv}$ dans une pièce d'environ $24 \mathrm{~m}^{3}$. Potentiellement, un purificateur comprenant des lampes UV consommant quelques dizaines de W devrait donc entraîner une diminution significative des concentrations des polluants de l'air ambiant puisque leur concentration est au plus de $100 \mathrm{ppbv}$ [10], même si la vitesse d'élimination dans le domaine des ppbv est plus faible que dans celui des ppmv ; en revanche, les concentrations ambiantes de $\mathrm{CO}$ et $\mathrm{CH}_{4}$ (quelques ppmv) ne pourront pas être abaissées d'une façon significative en raison du renouvellement de l'air.

Cette conclusion a conduit notre laboratoire à participer à un projet visant à la construction d'un prototype (brevet FR 2774914) destiné à la purification d'air dans des pièces individuelles. Ce prototype contenait des lampes d'une puissance électrique totale de $48 \mathrm{~W}$ et émettant autour de $365 \mathrm{~nm}$. $\mathrm{TiO}_{2}$ était supporté sur un matériau fibreux (environ $1300 \mathrm{~cm}^{2}$ ) de très faible épaisseur selon une méthode mise au point par Ahlstrom Paper Group (brevet PCT/FR99/00748). Le débit d'air était de $250 \mathrm{~m}^{3} \mathrm{~h}^{-1}$. L'efficacité de ce prototype a été évaluée dans une pièce ordinaire, non étanche, d'un volume de $83 \mathrm{~m}^{3}$, les analyses étant effectuées par GC après concentration des polluants par SPME (Solid Phase Micro-Extraction).[11]. 
Nous avons observé que les intensités des pics chromatographiques correspondant aux polluants collectés par SPME à l'aide d'une fibre recouverte de poly(diméthylsiloxane)carboxen sont très nettement diminuées lorsque les lampes UV du prototype, non optimisé, fonctionnent. Par exemple, les concentrations du benzène, du toluène et des xylènes sont abaissées d'un facteur 2 (Fig. 3 ).

Ces mesures sont, à notre connaissance, les premières effectuées avec de l'air ambiant dans une pièce ordinaire et non avec un seul polluant à concentration $10^{3}$ à $10^{5}$ fois supérieure dans une enceinte étanche de laboratoire. Elles démontrent clairement les possibilités de la photocatalyse en ce domaine.

\section{TRAITEMENT D'EFFLUENTS GAZEUX}

Pour les effluents industriels, il existe une multitude de situations. Donc, l'ordre de grandeur obtenu (10 $\mu \mathrm{mol} \mathrm{Wh}^{-1}$ ) en laboratoire ne peut donner une réponse générale, contrairement au cas de l'air ambiant, mais peut servir de guide. Il indique, par exemple, que pour éliminer un polluant à la concentration de 25 ppmv, il faudrait une puissance de l'ordre de $10 \mathrm{~kW}$ si le débit est de $100 \mathrm{~m}^{3} \mathrm{~h}^{-1}$. Ce type de calcul devrait permettre d'évaluer a priori si la photocatalyse peut être une solution réaliste pour tel ou tel cas.

En ce qui concerne les nuisances olfactives, la photocatalyse peut être une méthode élégante et intéressante si le composé malodorant possède un seuil olfactif de l'ordre du ppbv ou moins, et se trouve à une concentration elle-même assez faible qu'il s'agit d'abaisser au-dessous de ce seuil. Dans ce cas, la quantité de polluant à éliminer par unité de temps demeure assez faible si le débit n'est pas trop important.

Deux installations de grandes dimensions de purification d'air par photocatalyse au contact de $\mathrm{TiO}_{2}$ ont été construites en Amérique du Nord par Trojan et United Technologies pour éviter la pollution de nappes phréatiques par des hydrocarbures aliphatiques chlorés légers provenant de sites industriels abandonnés. Ces hydrocarbures sont extraits du sol et/ou de l'eau par stripage et l'air est ensuite traité par photocatalyse. Par exemple, pour une puissance de $19 \mathrm{~kW}$ et un débit d'air de l'ordre de $1500 \mathrm{~m}^{3} \mathrm{~h}^{-1}$, l'élimination du trichloroéthène, dont la concentration initiale est de l'ordre de $1000 \mathrm{ppmv}$, est supérieure à $99 \%$, malgré une humidité relative de $100 \%$. Le traitement photocatalytique s'avère économiquement plus intéressant que l'adsorption sur charbon actif. Cependant, il faut noter que le trichloroéthène est un cas favorable, car sa vitesse d'élimination est supérieure à l'ordre de grandeur que nous avons déterminé.

\section{Remerciements}

L'auteur remercie ses collaborateurs J. Disdier, C. Gaysse, G. Goutailler, C. Hoang-Van et D. Mas.

\section{Références}

1 P. Pichat, in Handbook of Heterogeneous Catalysis, edited by G. Ertl, H. Knözinger and J. Weitkamp (Wiley-VCH, Weinheim, 1997) vol. 4, pp. 2111-2122.

2. D.M. Blake, Bibliography of Work on the Photocatalytic Removal of Hazardous Compounds from Water and Air (NREL/TP-570-26797 Report, NTIS, Springfield, VA, 1999).

3. R. Armon, N. Laot, N. Narkis, J. Adv. Oxid. Technol. 3, 145 (1998).

4. D.M. Blake, P.-C. Maness, Z. Huang, E.J. Wolfrum, J. Huang, W.A. Jacoby, Separation Purif. Meth. 28, 1 (1999).

5. V. Roméas, P. Pichat, C. Guillard, T. Chopin, C. Lehaut, Ind. Eng. Chem. Res. 38, 3878 (1999) and refs therein.

6. M. Grätzel, Comments Inorg. Chem. 12, 93 (1991).

7. P. Pichat, Catalysis Today 19, 313 (1994).

8. L. Cermenati, C. Richter, A. Albini, Chem. Commun. 805 (1998).

9. P. Pichat, J. Disdier, C. Hoang-Van, D. Mas, G. Goutailler, C. Gaysse, Catalysis Today, sous presse.

10. J.J. Shah, H.B. Singh, Environ. Sci. Technol. 22, 1381 (1988).

11. J. Pawliszyn, Solid Phase Microextraction (Wiley-VCH, New York, 1997). 